\title{
Alternating-Phase Focusing for Dielectric-Laser Acceleration
}

\author{
Uwe Niedermayer, ${ }^{1, *}$ Thilo Egenolf, ${ }^{1}$ Oliver Boine-Frankenheim, ${ }^{1,3}$ and Peter Hommelhoff ${ }^{2}$ \\ ${ }^{1}$ Technische Universität Darmstadt, Schlossgartenstrasse 8, D-64289 Darmstadt, Germany \\ ${ }^{2}$ Department Physik, Friedrich-Alexander-Universität Erlangen-Nürnberg (FAU), Staudtstrasse 1, D-91058 Erlangen, Germany \\ ${ }^{3}$ GSI Helmholtzzentrum für Schwerionenforschung GmbH, Planckstrasse 1, D-64291 Darmstadt, Germany
}

(Received 13 June 2018; published 20 November 2018)

\begin{abstract}
The concept of dielectric-laser acceleration provides the highest gradients among breakdown-limited (nonplasma) particle accelerators. However, stable beam transport and staging have not been shown experimentally yet. We present a scheme that confines the beam longitudinally and in one transverse direction. Confinement in the other direction is obtained by a single conventional quadrupole magnet. Within the small aperture of $420 \mathrm{~nm}$ we find the matched distributions, which allow an optimized injection into pure transport, bunching, and accelerating structures. The combination of these resembles the photonics analogue of the radio frequency quadrupole, but since our setup is entirely two dimensional, it can be manufactured on a microchip by lithographic techniques. This is a crucial step towards relativistic electrons in the $\mathrm{MeV}$ range from low-cost, handheld devices and connects the two fields of attosecond physics and accelerator physics.
\end{abstract}

DOI: 10.1103/PhysRevLett.121.214801

Since dielectric-laser acceleration (DLA) of electrons was proposed in 1962 [1,2], the development of photonic nanostructures and the control of ultrashort laser pulses has advanced significantly (see Ref. [3] for an overview). Phase synchronous acceleration was experimentally demonstrated first in 2013 [4,5]. Damage threshold limited record gradients, more than an order of magnitude higher than in conventional accelerators, were achieved meanwhile for both relativistic [6] and low-energy electrons [7]. These gradients, so far, express themselves only in the generation of energy spread, not as a coherent acceleration. Moreover, the interaction length is limited to the Rayleigh length, after which the electron beam defocuses and hits the small (submicrometer) aperture. During synchronous acceleration, there are additional defocusing forces which cannot be overcome by magnetic focusing only [8] since equivalent magnetic focusing gradients would have to be in the MT/m range [9].

In this Letter we solve this outstanding problem with a laser-based scheme which allows transport and acceleration of electrons in dielectric nanostructures over arbitrary lengths. It is applicable to changing DLA period lengths, which is required to accelerate subrelativistic electrons. Moreover, we find the maximum tolerable emittances and beam envelopes in DLA beam channels. Another substantial advancement of our scheme is ballistic bunching of

Published by the American Physical Society under the terms of the Creative Commons Attribution 4.0 International license. Further distribution of this work must maintain attribution to the author(s) and the published article's title, journal citation, and DOI. subrelativistic electrons down to attosecond duration, while the beam remains transversely confined. Thus, our scheme makes DLA scalable, which paves the way for a low-cost accelerator on a microchip, providing electrons in the $\mathrm{MeV}$ range from a small-scale, potentially handheld device.

Our scheme uses only one spatial harmonic, namely, the synchronous one, but its magnitude and phase change along the DLA grating. This is interpreted as a time dependent focusing potential. A focusing concept using nonsynchronous spatial harmonics of traveling waves was presented by Naranjo et al. [10]. They derived stability due to retracting ponderomotive forces from the nonsynchronous spatial harmonics, while the synchronous one serves for acceleration. Our description is in the comoving real space, as compared to Naranjo's description in the spatial frequency domain. This supports changes of all grating-related quantities, while the Courant-Snyder (CS) theory [11] from conventional accelerator physics is still applicable. Stable beam confinement is achieved by alternating-phase focusing (APF), which had already been developed in the 1950s for ion acceleration [12-14]. However, the later developed radio frequency quadrupole (RFQ) cavities turned out to have better performance, especially for high current beams. Thus, APF was rejected in favor of the RFQ and was only rarely implemented [14]. In the 1980s APF was also proposed for grating-based linacs [15-18], but these three-dimensional designs are hardly feasible at optical wavelengths. Since 3D structures such as RFQs or rotated gratings are not feasible for lithographic fabrication on a microchip, we present an entirely two-dimensional APF scheme in this Letter, enabling stable and almost lossless electron transport in high-gradient DLA. 


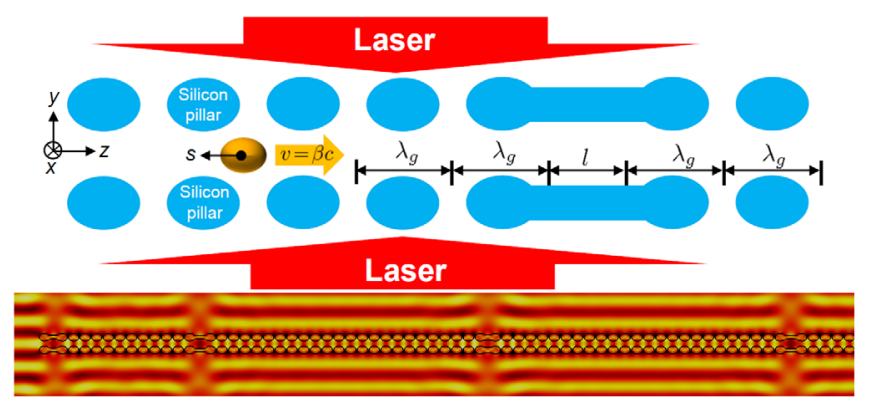

FIG. 1. (Top panel) Schematic view of a dual pillar DLA structure and a particle bunch around a reference particle and (bottom panel) simulation of the longitudinal time harmonic electric field.

We use standing wave dual pillar structures [7] as shown in Fig. 1, but our scheme can also be applied to Bragg cavity structures [19]. The $z$-polarized lasers, incident from both lateral sides, are modeled as plane waves with wavelength $\lambda_{0}=2 \mu \mathrm{m}$. In practice, they can be realized as pulsefront-tilted profiles [20-23] or on-chip wave guide systems [24]; see the Supplemental Material [9] for details. The tilted pulses appear to a single or a few electron bunches as a plane wave; however, the pulse duration impinging on each pillar is significantly reduced, and thus the damage threshold field strength is increased [25,26]. The Hamiltonian for single particle motion in the DLA is [27]

$$
H=\frac{1}{2 m_{e} \gamma}\left[p_{x}^{2}+p_{y}^{2}+\left(\Delta p_{z} / \gamma\right)^{2}\right]+V,
$$

where $\gamma=\left(1-\beta^{2}\right)^{-1 / 2}$ is the reference mass factor, $m_{e}$ the electron mass, $p_{x}, p_{y}$ the transverse momenta, and $\Delta p_{z}$ the deviation of longitudinal momentum from the reference particle at fixed laser phase (the black dot in Fig. 1). In [27] we showed by means of the Panofsky-Wenzel theorem [28] that the time dependent potential can be written as (see also the Supplemental Material [9])

$$
V=q \operatorname{Im}\left\{e_{1}\left[\frac{\lambda_{g}}{2 \pi} \cosh \left(\frac{\omega y}{\beta \gamma c}\right) e^{2 \pi i s / \lambda_{g}}-i s e^{i \varphi_{s}}\right]\right\},
$$

where $\omega=2 \pi c / \lambda_{0}$ is the laser angular frequency, $q$ is the (negative) electron charge, and $s$ is the distance of the particle behind the reference particle. The field strength of the resonant harmonic with the Wideroe condition $\lambda_{g}=\beta \lambda_{0}$ is $e_{1}$; i.e., with no loss of generality we work with the first (usually the strongest) spatial harmonic. The parameters $e_{1}, \varphi_{s}, \beta, \gamma$, and $\lambda_{g}$ are allowed to vary with the timelike cell index $n$. The synchronous phase $\varphi_{s}$ determines the energy gain of the reference particle as a function of the cell number (the acceleration ramp) as

$$
W_{\text {kin }}(N)=W_{\text {kin }, 0}+q \sum_{n=1}^{N} \lambda_{g}^{(n)} \operatorname{Re}\left\{e_{1}^{(n)} e^{i \varphi_{s}^{(n)}}\right\},
$$

where $W_{\text {kin, } 0}=83 \mathrm{keV}$ is the injection energy. The cell lengths increase according to the Wideroe condition as

$$
\frac{\lambda_{g}^{(n+1)}-\lambda_{g}^{(n)}}{\lambda_{0}}=\beta^{(n+1)}-\beta^{(n)}=\frac{q \lambda_{0} \operatorname{Re}\left\{e_{1}^{(n)} e^{i \varphi_{s}^{(n)}}\right\}}{m_{e} c^{2} \gamma^{(n)^{3}}} .
$$

For a given structure the synchronous phase is thus determined as $\varphi_{s}^{(n)}=\varphi_{0}-\arg \left(e_{1}\right)^{(n)}$, where

$$
\varphi_{0}=\arccos \left(\frac{m_{e} c^{2}}{q \lambda_{0}} \frac{\gamma^{3}}{\left|e_{1}\right|} \frac{\Delta \lambda_{g}}{\lambda_{0}}\right)
$$

In this Letter we use optimized structures which provide $\varphi_{0}$ independent of $n$ at an arbitrary chirp parameter $\Delta \lambda_{g}$, such that the synchronous phase $\varphi_{s}$ can be switched by a particular drift from one grating segment to another. Tying the phase $\arg \left(e_{1}\right)^{(n)}$ to $\lambda_{g}^{(n)}$ does not avoid a small drift in the normalized amplitude $\left|e_{1}^{(n)} / E_{L}\right| \approx 0.34 \ldots 0.39$ (see the Supplemental Material [9]), which is taken into account in the ramp [Eq. (3)].

Earnshaw's theorem dictates that constant focusing cannot be achieved in all three spatial directions simultaneously [29]. Thus, at least two focusing directions have to be alternating. In conventional Alvarez linacs or in synchrotrons constant focusing is applied in the longitudinal direction and alternating quadrupole lattices provide transverse confinement [30]. In our APF scheme, we apply the alternation to the disjoint focusing phase ranges of the longitudinal plane and the noninvariant transverse plane (y). Jumping the reference particle by means of a fractional cell drift between the orange circles in Fig. 2 provides stable transport at constant energy, and between the red dots we additionally obtain acceleration. The strong acceleration defocusing in $y$ is compensated by acceleration focusing at the longitudinally unstable phase. In the invariant $x$ direction a single conventional quadrupole magnet [9] suffices to confine the beam to an area in the center of the structure height, where the laser fields are homogeneous, i.e., do not depend on $x$.

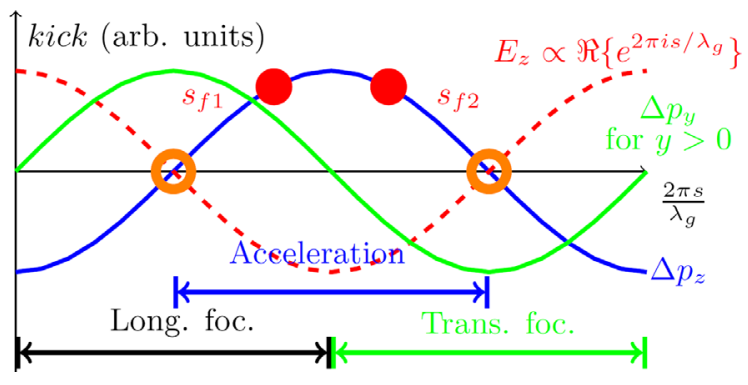

FIG. 2. Overview of electron acceleration and focusing properties as a function of phase. The circles denote the fixed points for different $\varphi_{s}$. 
We find the fixed points of the motion by setting $\nabla V=0$ as $s_{f 1}=\varphi_{s} \lambda_{g} / 2 \pi$ and $s_{f 2}=-\lambda_{g} / 2 \pi\left[\varphi_{s}+2 \arg \left(e_{1}\right)\right]$ and define $\Delta s_{1}=s-s_{f 1}$ and $\Delta s_{2}=s-s_{f 2}$. Note that in the longitudinal plane for $\arg \left(e_{1}\right)=0$ the fixed point $s_{f 1}$ is elliptic and $s_{f 2}$ is hyperbolic, and vice versa in the transverse plane. Expanding $V$ to second order and omitting constant terms shows the APF principle:

$$
\begin{array}{r}
V\left(x, y, s=s_{f 1}+\Delta s\right)=-V\left(x, y, s=s_{f 2}+\Delta s\right) \\
\quad=\frac{q\left|e_{1}\right| \lambda_{g}}{2 \pi}\left[\frac{1}{2}\left(\frac{\omega y}{\beta \gamma c}\right)^{2}-\frac{1}{2}\left(\frac{2 \pi}{\lambda_{g}} \Delta s\right)^{2}\right] \sin \left(\varphi_{0}\right) ;
\end{array}
$$

i.e., switching between $s_{f 1}$ and $s_{f 2}$ with $\Delta s=\Delta s_{1}=\Delta s_{2}$ flips the sign of the potential. Only the nonaccelerating case $\left(\varphi_{0}=\pi / 2\right)$ provides two interchangeable buckets, whereas a $\pi$-shifted version of the accelerating bucket will be decelerating and unstable due to a mismatch with the ramp. Hill's equations of the linearized motion are found from Eqs. (1) and (5) as

$$
\begin{gathered}
y^{\prime \prime}+K y=0, \\
\Delta s^{\prime \prime}-K \Delta s=0,
\end{gathered}
$$

where $K=\left|q \omega e_{1} /\left(m_{e} \beta^{3} \gamma^{3} c^{3}\right)\right| \sin \left(\varphi_{s}\right)$. Note that linearization leads to decoupling of the nonlinear equations of motion, which are coupled due to Eq. (2). The segments between two phase shifts are enumerated by $P$ such that

$$
\arg \left(e_{1}\right)(P)= \begin{cases}0, & P \text { odd }, \\ 2 \varphi_{0}, & P \text { even }\end{cases}
$$

leads to a sign alternation in the focusing function $K$ in Eq. (6). In order to switch between the two fixed points we take short drift sections denoted by $l$ and model the lattice as thick lenses of lengths $L^{f}$ and $L^{d}$. Each lattice cell consists of two segments and has $p$ transverse focusing and $p$ transverse defocusing elements; thus, its length is given by $L=L^{f}+l^{f}+L^{d}+l^{d}$, where

$$
\begin{gathered}
L^{f}=\sum_{n=1}^{p} \lambda_{g}^{(n)}, \quad L^{d}=\sum_{n=p+1}^{2 p} \lambda_{g}^{(n)}, \\
l^{f}=\left(2 \pi-\varphi_{s}^{(p)}\right) \lambda_{g}^{(p)} / \pi, \quad l^{d}=\left(\pi-\varphi_{s}^{(2 p)}\right) \lambda_{g}^{(2 p)} / \pi .
\end{gathered}
$$

The solution to Eq. (6) is found by applying the CS formalism [11] to the channel of thick focusing $(F)$ and defocusing $(D)$ elements. We start with a nonaccelerating transport structure, i.e., $\varphi_{0}=\pi / 2$, where the lattice cells are strictly periodic. In a long lattice cell $(p \gg 1)$ we can neglect the drift sections and represent it as [9]

$$
\mathbf{M}(z, L)= \begin{cases}\mathbf{M}_{f}(z), & 0<z<L / 2, \\ \mathbf{M}_{d}(z-L / 2) \mathbf{M}_{f}(L / 2), & L / 2<z<L,\end{cases}
$$

with the length $L=(2 p+1) \lambda_{g}$. The phase advance per cell $\sigma$ is given for a strictly periodic $F D$-cell by

$$
\cos (\sigma)=\frac{1}{2} \operatorname{Tr}\{\mathbf{M}(L, L)\}=\cos \left(\frac{\sqrt{K} L}{2}\right) \cosh \left(\frac{\sqrt{K} L}{2}\right) .
$$

The CS parameters $\eta=(\hat{\beta}, \hat{\alpha}, \hat{\gamma})^{\mathrm{T}}$ are mapped from one point to another by the matrix $\mathbf{T}$ (see the Supplemental Material [9]) and fulfill the eigenvector relation $\eta_{e}=\mathbf{T} \eta_{e}$ for their initial values. For small $\sigma$ the constant $\hat{\beta}$ function in the smooth approximation is found from $\langle\hat{\beta}\rangle=L / \sigma$. However, the most critical issue in DLA is to match a given emittance to the tiny aperture. Thus, the maximum of the $\hat{\beta}$ function, which appears at $L / 4$, needs to be minimal (see Fig. 3). The only variable parameter in an experimental setup is the laser field strength. Its tuning range from maximal admissible beam size to the structure damage threshold $[25,26]$ is indicated by the black arrow. The evolution of the transverse phase space is shown below, where the particles were initially arranged on a Cartesian grid and only the longterm surviving ones are displayed in red. For simplicity, this simulation starts at $L / 4$ in order to avoid correlations in the conjugate variables. This plot uses zero bunch length, but stability is also attained for an unbunched beam; see the video in the Supplemental Material [9]. The blue ellipses indicate the strictly periodic linear case, which is slightly smaller in area due to the cosh potential in Eq. (2) being
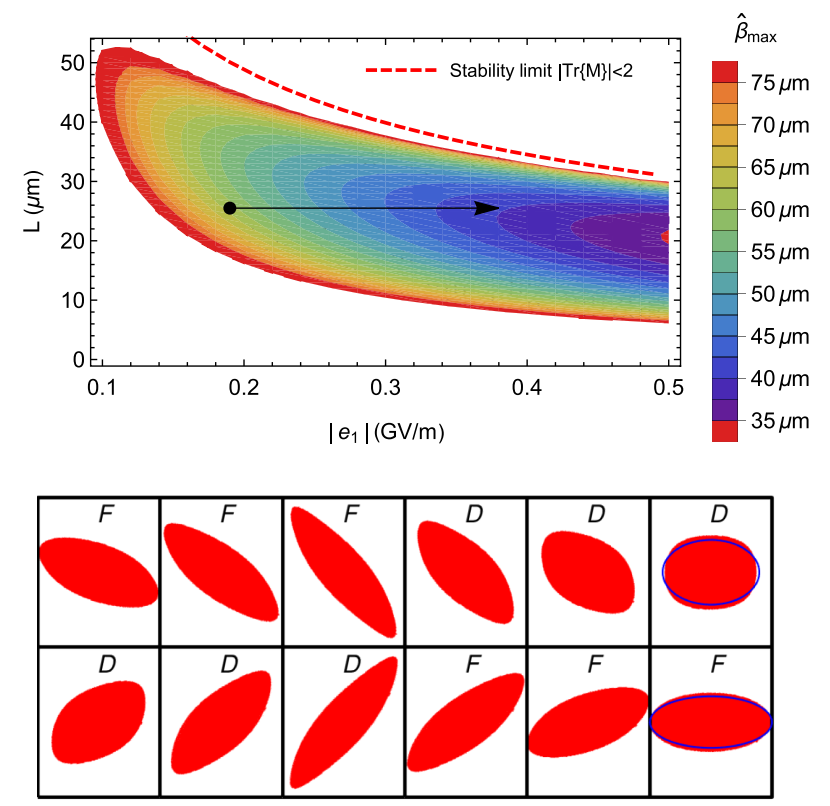

FIG. 3. (Top panel) Contours of $\hat{\beta}_{\max }=\hat{\beta}(L / 4)$ in the $\left(\left|e_{1}\right|, L\right)$ plane. The arrow indicates the laser amplitude dependent tuning range. (Bottom panel) The transverse phase space evolution (parameters at the black dot on top) of particles, not hitting the aperture $( \pm 0.21 \mu \mathrm{m})$ within 1200 DLA cells, is shown as every two DLA cells. The blue ellipses are the linear theory, at minimum and maximum beam size. 


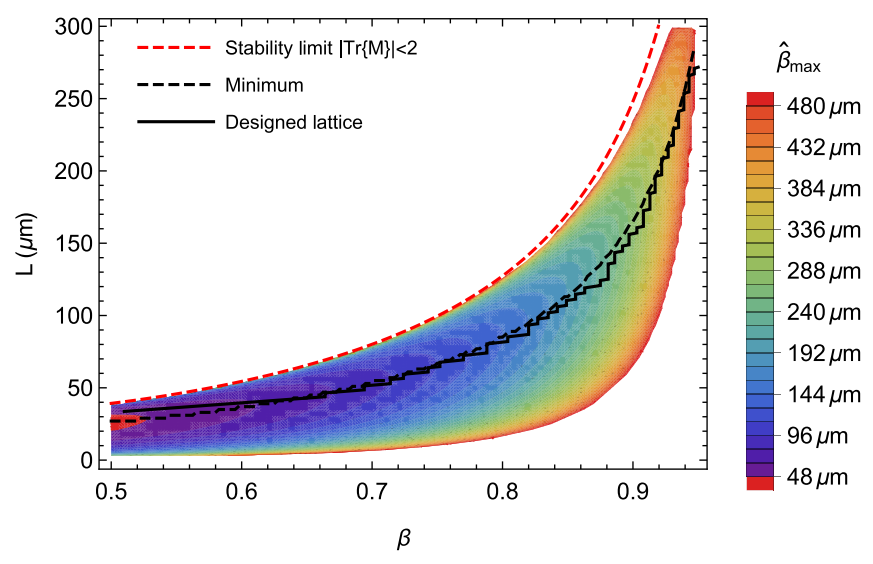

FIG. 4. Contours of $\hat{\beta}_{\max }=\hat{\beta}(L / 4)$ in the $(\beta, L)$ plane. The designed accelerator lattice is a trade-off between following the minimum and minimizing the mismatch at the jumps.

steeper than the square well in Eq. (5). In the linear case the single particle emittances are invariants

$$
\begin{gathered}
\varepsilon\left(y, y^{\prime}\right)=\hat{\gamma} y^{2}+2 \hat{\alpha} y y^{\prime}+\hat{\beta} y^{\prime 2}, \\
\varepsilon_{L}\left(\Delta s, \Delta s^{\prime}\right)=\hat{\gamma}_{L} \Delta s^{2}+2 \hat{\alpha}_{L} \Delta s \Delta s^{\prime}+\hat{\beta}_{L} \Delta s^{\prime 2},
\end{gathered}
$$

where $\Delta s^{\prime}=\Delta W /\left(m_{e} \gamma^{3} \beta^{2} c^{2}\right)$, and we introduce longitudinal CS functions as a half lattice cell shift of the transverse ones, $\eta_{L}(z)=\eta(z-L / 2)$.

An accelerating lattice can be attained by taking the initial values from the eigenvalue solution and successively multiplying the segment maps as $\eta_{N}=\mathbf{T}_{N} \ldots \mathbf{T}_{1} \eta_{e}$ to it. In nonperiodic lattices the longitudinal CS functions have to be calculated individually with the same procedure. If the change in length from one period to another is small, the $\hat{\beta}$ function can be approximated by the eigenvalue solution in each cell, which is, however, discontinuous at the boundaries. The line of increasing minimum of $\hat{\beta}_{\max }$ in Fig. 4 is followed only approximately. The increase is counteracted by

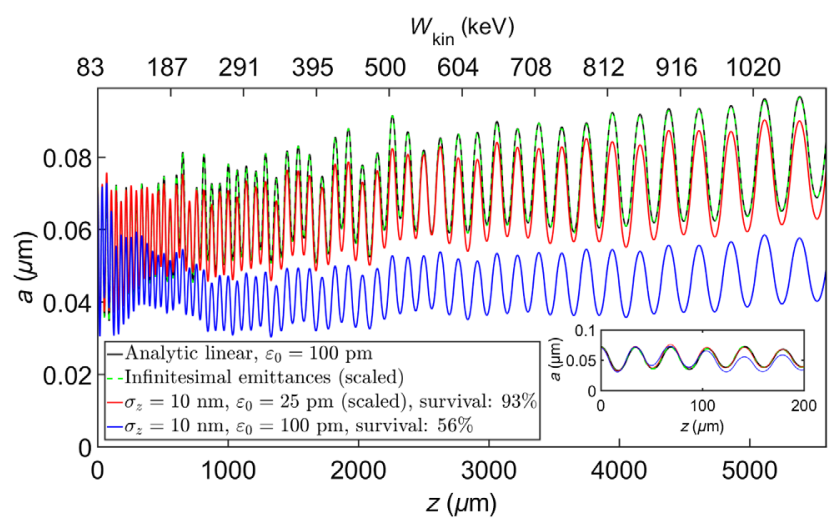

FIG. 5. Analytical [Eq. (10)] and numerical (rms) beam envelopes, scaled to identical initial beam size at $\varepsilon=100 \mathrm{pm}$. (Inset) Enlargement of the beginning. adiabatic emittance damping due to momentum conservation. Altogether the beam envelope can be written as [30]

$$
a(z)=\sqrt{\hat{\beta}(z) \frac{\varepsilon_{0} \beta_{0} \gamma_{0}}{\beta(z) \gamma(z)}},
$$

where the 0 indices denote initial values. Acceleration from $83 \mathrm{keV}$ to $1 \mathrm{MeV}$ at $\varphi_{0}=4 \pi / 3$, with an average gradient of $187 \mathrm{MeV} / \mathrm{m}$ and $500 \mathrm{MV} / \mathrm{m}$ incident laser field strength from both sides, is shown to be well confined within the physical aperture of $\pm 0.21 \mu \mathrm{m}$ in Fig. 5. The analytical and numerical results coincide for infinitesimally low emittance. At small but achievable emittances [31,32], we obtain 56\% transmission for $\varepsilon_{0}=100 \mathrm{pm}$ (see the video in the Supplemental Material [9]), and $93 \%$ for $\varepsilon_{0}=25 \mathrm{pm}$. The phase space density at top energy is plotted in Fig. 6, where $\Phi_{P}$ and $\Delta W$ are the longitudinal coordinates in the comoving (Galilean) laboratory frame. As in Fig. 3, the initial particle positions in Fig. 6 (left panel) are arranged on a Cartesian grid, and only the ones that make it to $1 \mathrm{MeV}$ are drawn in red. The blue ellipse corresponds to an initially matched bunch adjusted to the area of the surviving particles. Note that this size is slightly reduced at finite transverse emittance; thus, we choose $\sigma_{z}=10 \mathrm{~nm}$. Below this bunch length the transmission depends only on the initial transverse emittance, i.e., is fully scalable.

The APF scheme discussed here can also be used to attain the bunching needed to inject into the accelerator structure. Creating and removing sinusoidal energy spread (see the Supplemental Material [9] and the video therein) results in extremely short (attosecond) bunch lengths at acceptably low energy spread. The phase alternation additionally provides transverse confinement, which can be matched to the initial CS functions of the accelerator. The particles not captured are defocused, while the captured ones remain at small longitudinal and transverse amplitudes, within the limits of Liouville's theorem. The phase
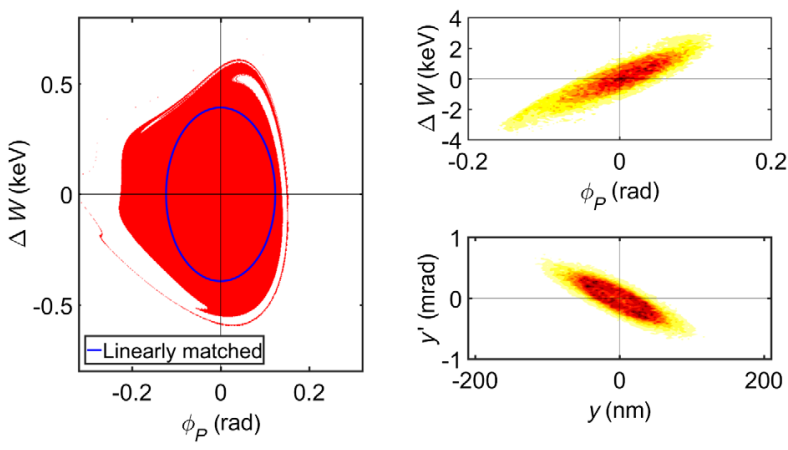

FIG. 6. (Right panels) Phase space after acceleration of a Gaussian bunch up to $1 \mathrm{MeV}$ and (left panel) transmittable initial longitudinal distribution at $83 \mathrm{keV}$ for $y=y^{\prime}=0$. The blue ellipse represents a linearly matched bunch with a total bunch length $4 \sigma_{z}=40 \mathrm{~nm}$. 


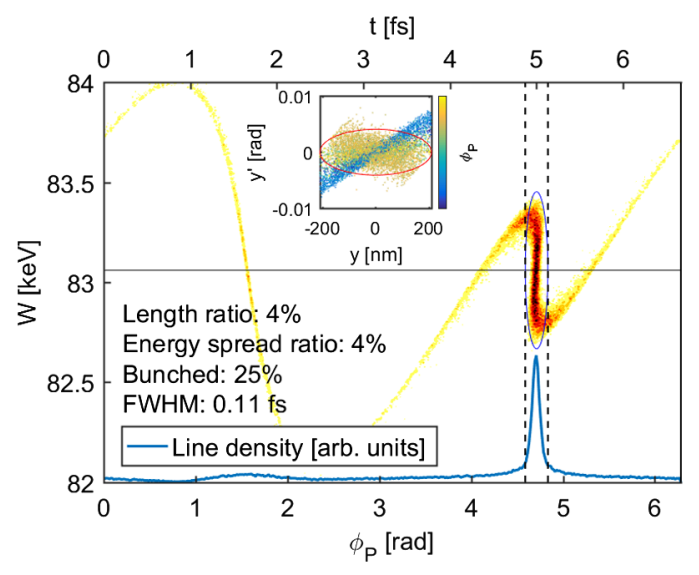

FIG. 7. Periodic longitudinal phase space after APF bunching. The initial beam parameters for the accelerator given by the ellipses are met. The small plot shows the transverse phase space, where the particle phase is color coded.

space after the buncher is plotted in Fig. 7. Both ellipses are matched for the injection into the accelerator (the blue ellipses in Figs. 7 and 6 are identical). Before the buncher the energy spread is $\sigma_{\Delta W}=16 \mathrm{eV}$. The initial longitudinal emittance equals the final one in the ellipse which has $25 \%$ of the particles captured. The duration is decreased to $4 \%$ $(\approx 260$ as), whereas the energy spread is increased by the same ratio. The initial CS functions are determined by inverse mapping of the desired final values for the accelerator. Additionally to the injection into DLAs, these short bunches are also very promising for ultrafast time-resolved electron microscopy.

In conclusion, we developed a scheme that makes DLA fully scalable. We showed for the first time how a focusing lattice, which relies on APF only, can be integrated. The entire accelerator or parts such as a single focusing stage or the buncher can now be experimentally approached. Acceleration of sub- $100 \mathrm{keV}$ electrons from readily available sources up to the $\mathrm{MeV}$ range with gradients of several $100 \mathrm{MeV} / \mathrm{m}$ works with transmission rates well above $90 \%$. The admissible synchronous phase is determined by the available bunch length at injection. We showed that controlling this nonlinear dependence is crucial to avoiding particle loss. Our bunching scheme provides the required attosecond bunches with the matched energy spread and a reasonable capture rate of $25 \%$. In principle, fully adiabatic bunching as in the RFQ is also possible. This would, however, require a larger total length. The APF scheme can also be scaled to higher energies, where smaller beam size and larger physical apertures due to longer roll-off of the evanescent acceleration fields will ease the requirements.

U.N. would like to thank Holger Podlech for the discussions on APF. This work is funded by the Gordon and Betty Moore Foundation (Grant No. GBMF4744) and the German Federal Ministry of Education and Research (Grant No. FKZ: 05K16RDB). *niedermayer@temf.tu-darmstadt.de

[1] K. Shimoda, Appl. Opt. 1, 33 (1962).

[2] A. Lohmann, IBM Technical Note 5, 169, 1962.

[3] R. J. England et al., Rev. Mod. Phys. 86, 1337 (2014).

[4] E. A. Peralta, K. Soong, R. J. England, E. R. Colby, Z. Wu, B. Montazeri, C. McGuinness, J. McNeur, K. J. Leedle, D. Walz, E. B. Sozer, B. Cowan, B. Schwartz, G. Travish, and R. L. Byer, Nature (London) 503, 91 (2013).

[5] J. Breuer and P. Hommelhoff, Phys. Rev. Lett. 111, 134803 (2013).

[6] K. P. Wootton, Z. Wu, B. M. Cowan, A. Hanuka, I. V. Makasyuk, E. A. Peralta, K. Soong, R. L. Byer, and R. J. England, Opt. Lett. 41, 2696 (2016).

[7] K. J. Leedle, A. Ceballos, H. Deng, O. Solgaard, R. F. Pease, R. L. Byer, and J. S. Harris, Opt. Lett. 40, 4344 (2015).

[8] A. Ody, P. Musumeci, J. Maxson, D. Cesar, R. J. England, and K. P. Wootton, Nucl. Instrum. Methods Phys. Res., Sect. A 865, 75 (2017).

[9] See Supplemental Material at http://link.aps.org/ supplemental/10.1103/PhysRevLett.121.214801 for mathematical/technical details and movies.

[10] B. Naranjo, A. Valloni, S. Putterman, and J. B. Rosenzweig, Phys. Rev. Lett. 109, 164803 (2012).

[11] E. Courant and H. Snyder, Ann. Phys. (N.Y.) 3, 1 (1958).

[12] I. B. Fainberg, in Proceedings of the 1st International Conference on High-Energy Accelerators (HEACC), Geneva, 1956, edited by E. Regenstreif (CERN, Geneva, 1956).

[13] P. Lapostolle, Los Alamos Technical Report No. 11601, 1989.

[14] T. P. Wangler, RF Linear Accelerators (Wiley-VCH, Weinheim, 2008).

[15] R. B. Palmer, Part. Accel. 11, 81 (1980).

[16] R. B. Palmer, AIP Conf. Proc. 91, 179 (1982).

[17] K. Kim and N. Kroll, Lawrence Berkeley Laboratory Report No. LBL-14378, 1982.

[18] M. Pickup, Report No. CLNS-85/655, 1985.

[19] U. Niedermayer, O. Boine-Frankenheim, and T. Egenolf, J. Phys. Conf. Ser. 874, 012041 (2017).

[20] Y. Wei, M. Ibison, G. Xia, J. D. A. Smith, and C. P. Welsch, Appl. Opt. 56, 8201 (2017).

[21] D. D. Cesar, J. Maxson, X. Shen, K. P. Wootton, S. Tan, R. J. England, and P. Musumeci, Nucl. Instrum. Methods Phys. Res., Sect. A (to be published).

[22] D. Cesar, J. Maxson, X. Shen, K. P. Wootton, S. Tan, R. J. England, and P. Musumeci, Opt. Express 26, 29216 (2018).

[23] M. Kozák, J. McNeur, N. Schönenberger, J. Illmer, A. Li, A. Tafel, P. Yousefi, T. Eckstein, and P. Hommelhoff, J. Appl. Phys. 124, 023104 (2018).

[24] T. W. Hughes, S. Tan, Z. Zhao, N. V. Sapra, K. J. Leedle, H. Deng, Y. Miao, D. S. Black, O. Solgaard, J. S. Harris, J. Vuckovic, R. L. Byer, S. Fan, R. J. England, Y. J. Lee, and M. Qi,Phys. Rev. Applied 9, 054017 (2018).

[25] P. P. Pronko, P. A. Van Rompay, C. Horvath, F. Loesel, T. Juhasz, X. Liu, and G. Mourou, Phys. Rev. B 58, 2387 (1998).

[26] K. Soong, R. L. Byer, E. R. Colby, R. J. England, and E. A. Peralta, AIP Conf. Proc. 1507, 511 (2012). 
[27] U. Niedermayer, T. Egenolf, and O. Boine-Frankenheim, Phys. Rev. Accel. Beams 20, 111302 (2017).

[28] W. K. H. Panofsky and W. A. Wenzel, Rev. Sci. Instrum. 27, 967 (1956).

[29] S. Earnshaw, Trans. Cambridge Philos. Soc. 7, 97 (1842).

[30] S. Y. Lee, Accelerator Physics (World Scientific, Singapore, 2004).
[31] D. Ehberger, J. Hammer, M. Eisele, M. Krüger, J. Noe, A. Högele, and P. Hommelhoff, Phys. Rev. Lett. 114, 227601 (2015).

[32] A. Feist, N. Bach, N. Rubiano da Silva, T. Danz, M. Möller, K. E. Priebe, T. Domröse, J. G. Gatzmann, S. Rost, J. Schauss, S. Strauch, R. Bormann, M. Sivis, S. Schäfer, and C. Ropers, Ultramicroscopy 176, 63 (2017). 